\section{Post-replication repair: ubiquitin throws the switch}

After DNA damage, the replication machinery recruits specialized bypass polymerases to stalled replication forks. This allows the machinery to proceed beyond lesions that remain in the DNA. In their recent paper, Lehmann and colleagues (Mol. Cell 14, 491-500; 2004) suggest that mono-ubiquitination of PCNA - a factor essential for replication-linked repair and normal replication — might trigger the switch from a conventional replicative polymerase to a bypass polymerase.

Earlier work in yeast established that after DNA damage, PCNA is mono-ubiquitinated on a single lysine residue (Lys 164) by the Rad18 ubiquitin E3 ligase (Nature 419, 135-141; 2002). Human PCNA also seems to be ubiquitinated at this conserved lysine residue in response to DNA damage. In the new work, the authors extend these earlier findings and show that mono-ubiquitination of human PCNA also requires the Rad18 protein. However, unlike yeast PCNA, which is also modified at the same lysine (by Lys 63-linked polyubiquitin chains), polyubiquitinated human PCNA could not be detected. This may reflect a divergence in function between yeast and human PCNA. Alternatively, polyubiquitinated human PCNA may be more prone to de-ubiquitination or degradation, thus rendering it a more elusive species in human cells than in yeast.

The next step was to try and pin down the functional consequences of this modification. Once again, important clues for unravelling function came from earlier work. First, work from the authors (Genes Dev. 15, 158-172; 2001) showed colocalization between PCNA and a bypass polymerase, pol $\eta$, to stalled replication forks after ultraviolet irradiation. Second, epistasis analysis in yeast demonstrated that a ubiquitination-deficient PCNA-K164R mutant and the yeast poln function in the same pathway (Nature 425, 188-191; 2003). Therefore, pol $\eta$ emerged as a possible candidate for binding to mono-ubiquiti-

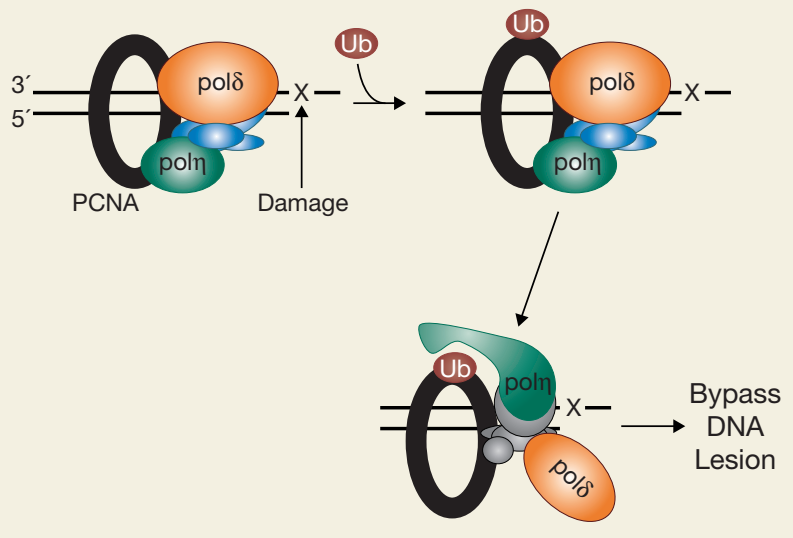

Binding of pol $\eta$ to monoubiquitinated PCNA helps bypass DNA lesions.

nated PCNA. Indeed, the authors of the new study found that poln colocalized with ubiquitinated PCNA at sites of stalled DNA replication, and the two proteins co-immunoprecipitated after exposure to damage-inducing agents. Next, they defined two motifs in pol $\eta$ that are required for binding to PCNA, including a motif related to the CUE domain that is found in many ubiquitin-binding proteins. Finally, a mutant lacking these motifs failed to rescue the sensitivity of poln-deficient strains to DNA damage, demonstrating that this interaction is physiologically relevant to DNA repair.

The authors propose an attractive model, in which in situ monoubiquitination of PCNA enhances its affinity for pol $\eta$ and triggers the switch from replication to a bypass mechanism at damage sites. Other bypass polymerases also interact with PCNA in vitro; thus, it will be interesting to determine whether these polymerases interact with ubiquitinated PCNA and, if so, whether they cooperate with pol $\eta$.

SOWMYA SWAMINATHAN 